\title{
Radiation Testing of PICA at the Solar Power Tower
}

\author{
Susan White ${ }^{1}$ \\ NASA Ames Research Center, Moffett Field, CA 94035
}

\begin{abstract}
Sandia National Laboratory's Solar Power Tower was used to irradiate specimens of Phenolic Impregnated Carbon Ablator (PICA), in order to evaluate whether this thermal protection system material responded differently to potential shock layer radiative heating than to convective heating. Tests were run at 50,100 and $150 \mathrm{~W} / \mathrm{cm}^{2}$ levels of concentrated solar radiation. Experimental results are presented both from spectral measurements on 1$10 \mathrm{~mm}$ thick specimens of PICA, as well as from in-depth temperature measurements on instrumented thicker test specimens. Both spectral measurements and measured in-depth temperature profiles showed that, although it is a porous, low-density material, PICA does not exhibit problematic transparency to the tested high levels of NIR radiation, for all pragmatic cm-to-inch scale thicknesses. PICA acted as a surface absorber to efficiently absorb the incident visible and near infrared incident radiation in the top 2 millimeter layer in the Solar Power Tower tests up to $150 \mathrm{~W} / \mathrm{cm}^{2}$.
\end{abstract}

\section{Nomenclature}

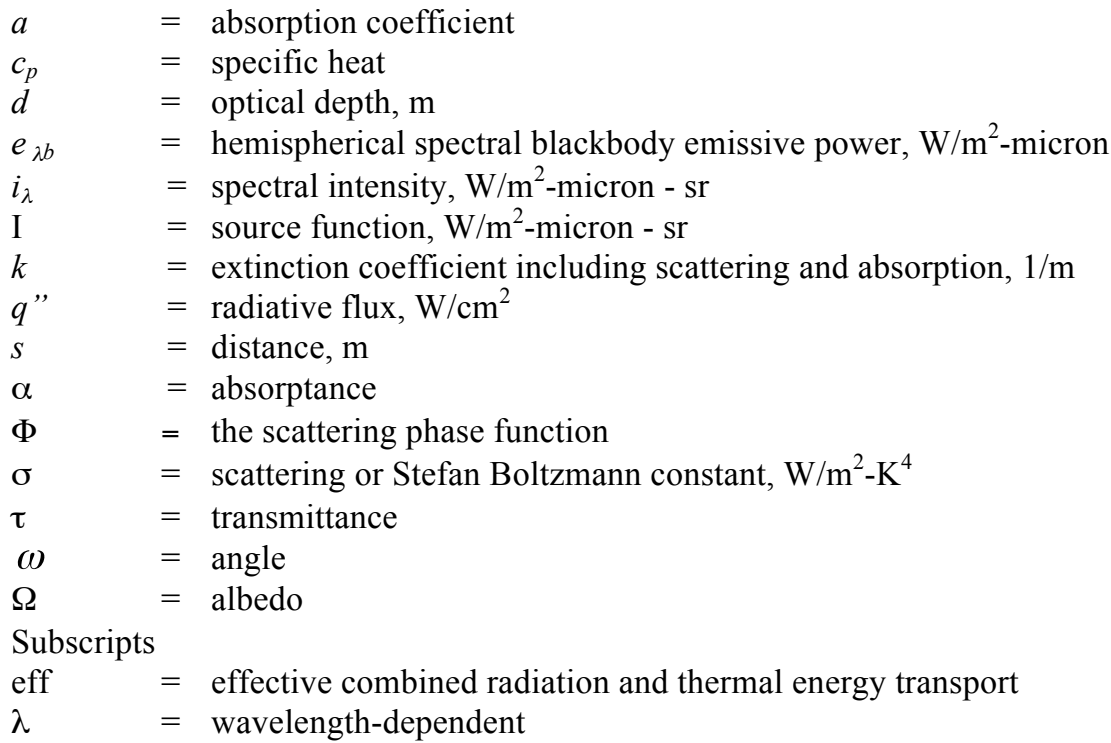

\section{Introduction}

spacecraft entering or traveling through an atmosphere at very high speed is typically subjected to a high heat A flux and requires a thermal protection system (TPS) to withstand this type of extreme environment. The higher the vehicle's relative speed, the greater the role shock-layer radiation plays in the overall heat flux. Developing a TPS to mitigate high levels of radiative heat flux is generally very different than designing a TPS to handle high heat loads from environments dominated by convective heating, primarily because the physics of the energy

\footnotetext{
${ }^{1}$ Research Scientist, Thermal Protections Materials and Systems Branch, MS 234-1. NASA Ames Research Center, Moffett Field, CA 94035. 
transport is so different. Most high density thermal protection materials act like surface absorbers, which absorb or block radiation fully at the surface. However, in low density porous TPS materials, the potential for in-depth transmission and in-depth absorption of radiation is a concern to vehicle designers and needs further evaluation. For higher radiation flux trajectories, radiation transmission could potentially lead to excess in-depth absorption, pyrolysis and/or bondline heating in TPS materials.

The spectral distribution of shock-layer radiation is governed by the gas composition, temperature and pressure. For air, shock-layer radiation is concentrated in narrow lines in the Near-Infrared (NIR) and in the Vacuum Ultraviolet (VUV) regions of the electromagnetic spectrum, due to air thermochemistry. In contrast, shock-layer radiation at Saturn's moon Titan is dominated by Ultraviolet lines.

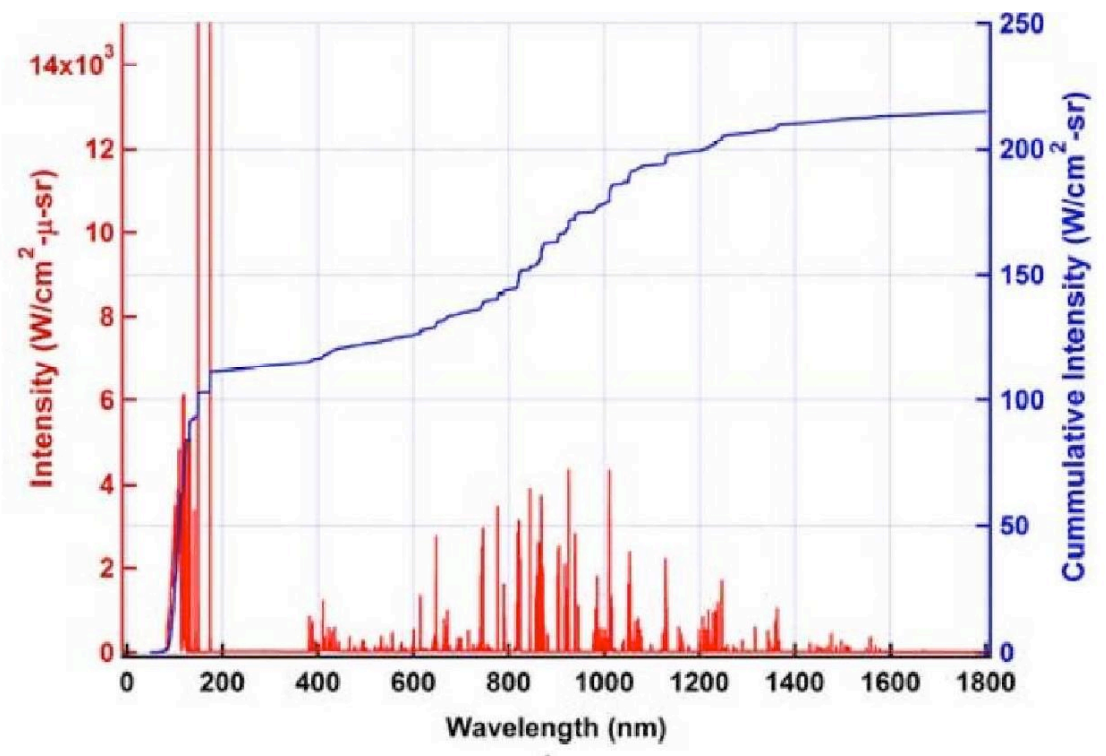

Figure 1. Predicted spectrum of radiative flux to CEV vehicle outer moldline

Figure 1 shows predicted wavelength distribution and cumulative level of radiative heating for lunar return to the CEV outer moldline, from Bose ${ }^{1}$ et al. ${ }^{1}$ The predicted radiation spectrum shown is concentrated in the vacuum ultraviolet (VUV) and the near infrared (NIR) regions. Testing was separated into these two VUV and NIR regimes, which require different setups, radiation sources, and detectors. No VUV transmission was measured in this material, either in direct transmission ${ }^{2}$ or using a VUV integrating sphere ${ }^{3}$. VUV spectrometers were used since no high-powered non-pulsed sources of VUV radiation were available for testing. This paper describes near-infrared (NIR) spectrometry and related measurements performed at the Solar Power Tower, which is described in the next section. 


\section{Testing Facility and Setup}

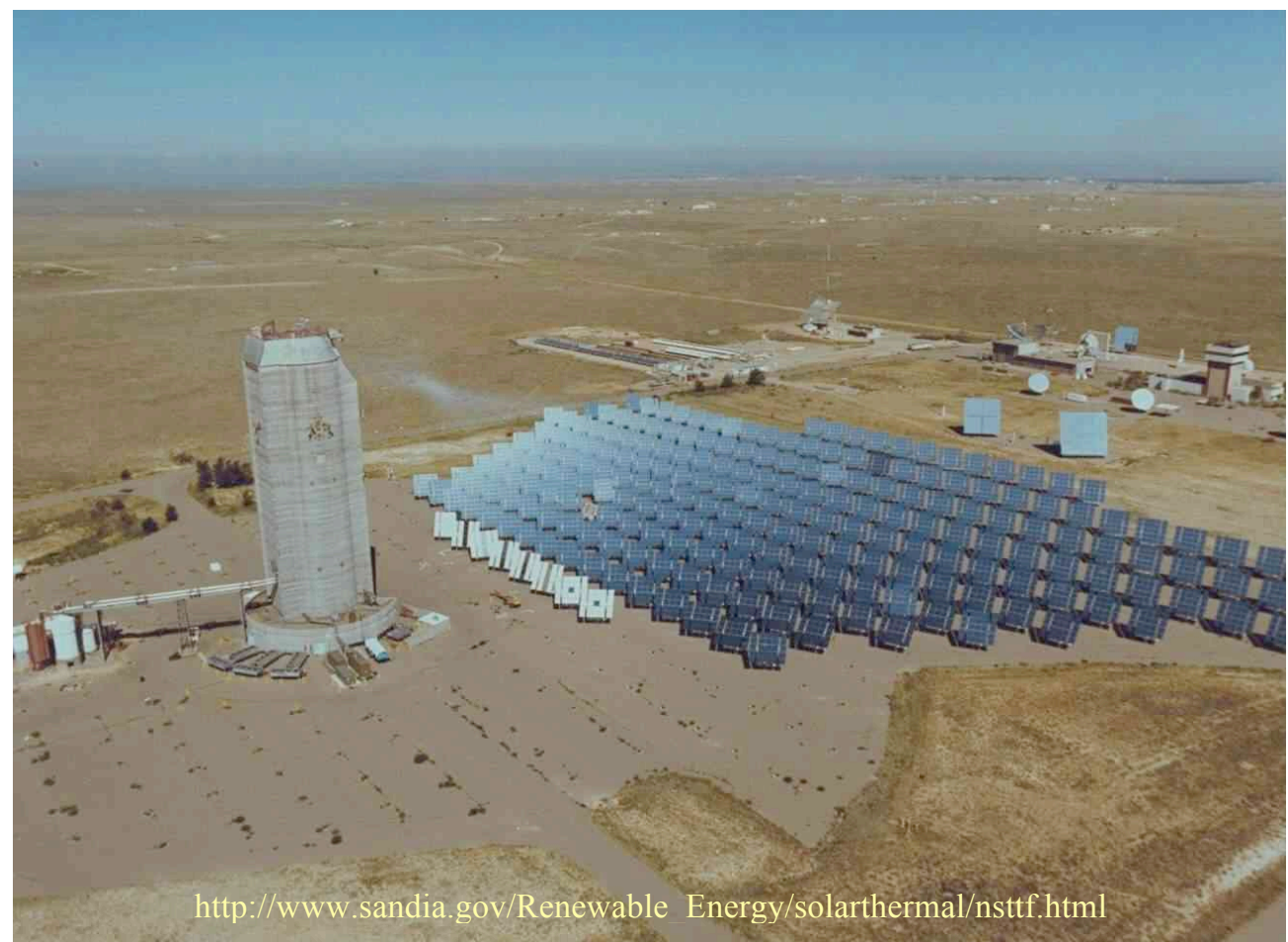

Figure 2. The Solar Power Tower uses an array of heliostat mirrors to focus solar radiation on the test target located at the top of the tower or the wind tunnel test bay.

Figure 2 is an aerial photo of the Sandia National Lab's Solar Power Tower at Kirtland Air Force Base in Albuquerque, New Mexico. The Solar Power Tower setup at the time of this testing provided pure non-pulsed radiation fluxes up to $200 \mathrm{~W} / \mathrm{cm}^{2}$ over large test areas, on the scale of 1 meter in diameter, for long durations.

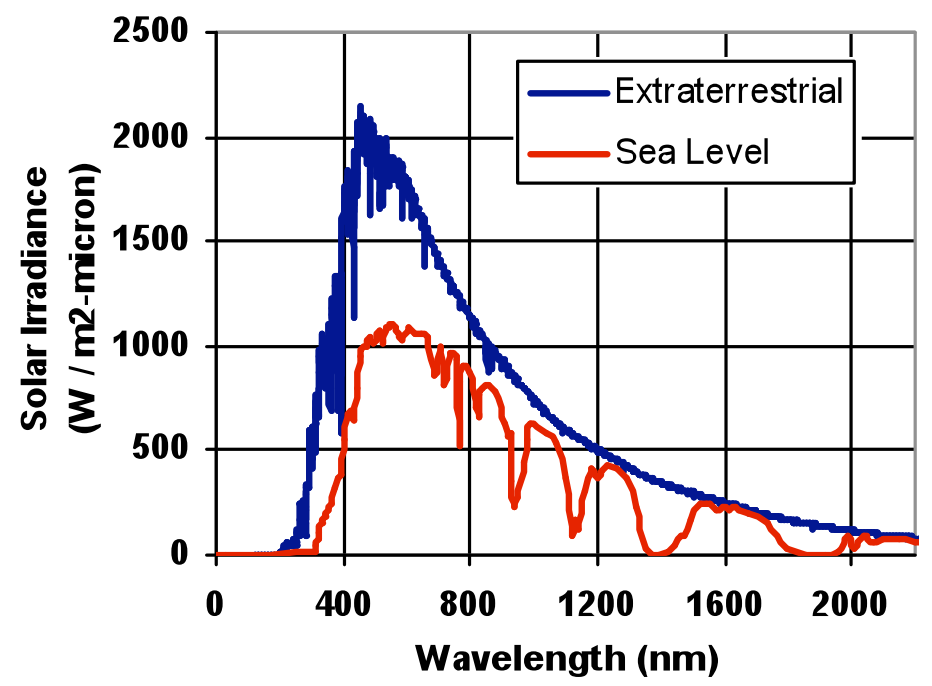

Figure 3. Solar radiation reference values from ASTM G159-98 and ASTM E-490 
Figure 3 shows a plot of solar radiation, according to ASTM G159-98 and ASTM E-490 standards. Solar radoiation includes high levels of visible and NIR radiation, in spite of water absorption bands, but due to strong atmospheric VUV absorption, terrestrial solar collectors cannot be used for VUV radiation testing. The absorption band at $1380 \mathrm{~nm}$ was used to quantify secondary effects except when successfully removed using a cold filter or a specific spectral filter.

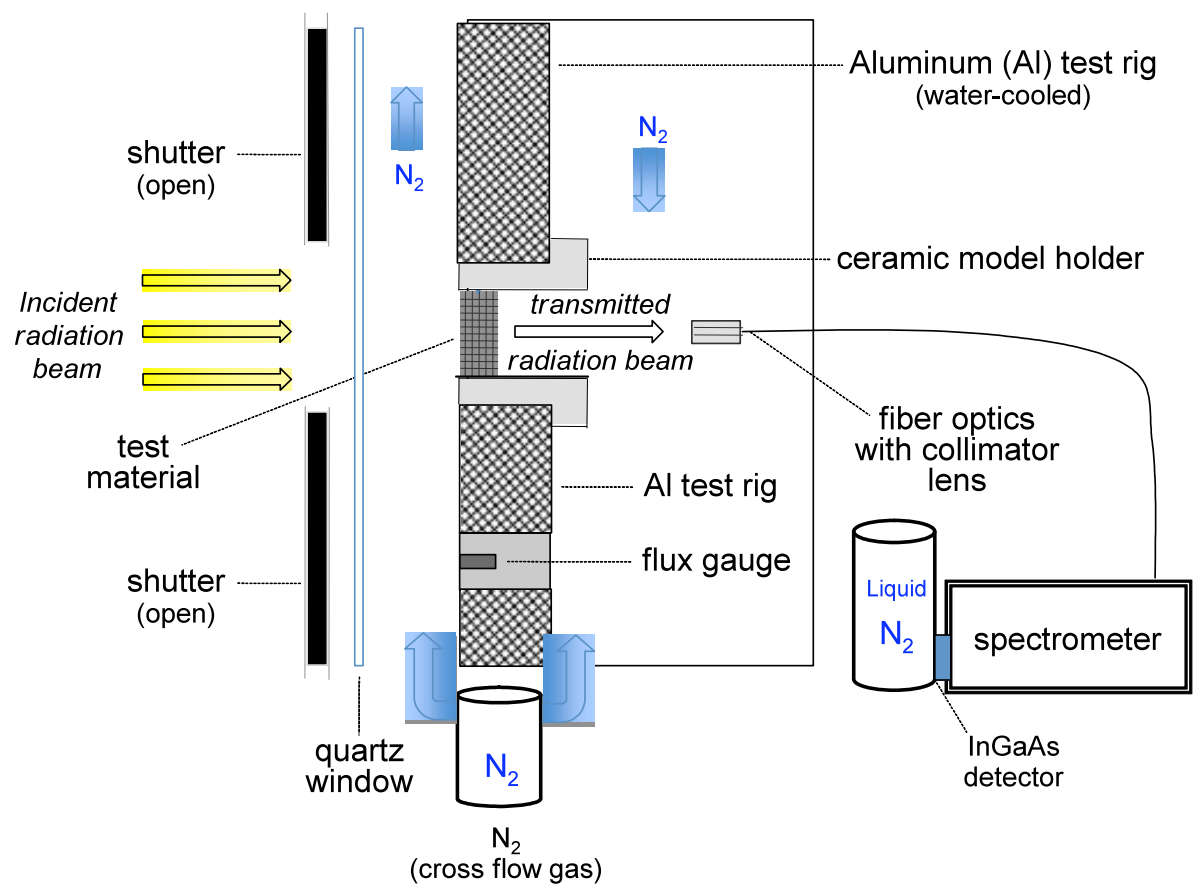

Figure 4. Schematic of the test setup used at Sandia

Figure 4 shows the setup used for spectrometer measurements as previously described ${ }^{4}$ for tests made on TPS materials in the Solar Power Tower. The external shutter in front of the quartz window protected the test material during the 15-second flux ramp-up time required for the heliostat array to attain the test flux level, using the heliostat controllers installed at the facility at that time. The external shutter was upgraded for these tests for precisely controlled and repeatable 1-second exposures of the thin 1-10 $\mathrm{mm}$ thick test specimens, as well as for longer durations, such as 55 seconds, for thicker thermocouple instrumented models. The Solar Tower facility's Kendell radiometer was used to establish radiation flux condition before testing, and the calibrated Kendell measurements were made pre- and post-test for each exposure. The spectrometer timing was synchronized with the shutter by a trigger to the spectrometer's data acquisition system.

The test material itself, typically a thin $2 \mathrm{~mm}$ specimen of test material, was positioned normal to the incident flux. The test material was held at the edges to allow direct viewing of the back face of the exposed area. A crossflow of nitrogen gas was continuously blown across the test material's front face, in order to reduce beam blockage by pyrolysis gas or soot as well as oxidation. The test material was held fixed in a coated ceramic LI-2200 sample holder, in order to thermally and electrically isolate the test model from the water-cooled aluminum framed test rig. A local flux gauge measured the flux during each test exposure to record potential flux variations between calibrated measurements. The portable spectrometer used was a Princeton Instruments / Acton Spectrapro $2300 \mathrm{i} 0.3 \mathrm{~m}$ focal length, triple-grating spectrometer. For these infrared measurements, a liquid Nitrogen cooled Indium Gallium Arsenide (InGaAs) linear array detector was used with a $150 \mathrm{~g} / \mathrm{mm}$ grating to capture spectra over the wavelength ranges from 0.85 to 1.2 or 1.1 to 1.6 microns. Typically ten to twelve spectral scans were completed per second, even for a $10 \mathrm{msec}$ integration time including the time required for positioning the grating.

In order to accurately convert the data from raw counts to flux units, the wavelength-dependent response curve of the test equipment was measured. The response was calibrated using the NIST-traceable spectral output of the SphereOptics Integrating Sphere in the NASA Ames E.A.S.T. facility ${ }^{5}$ 


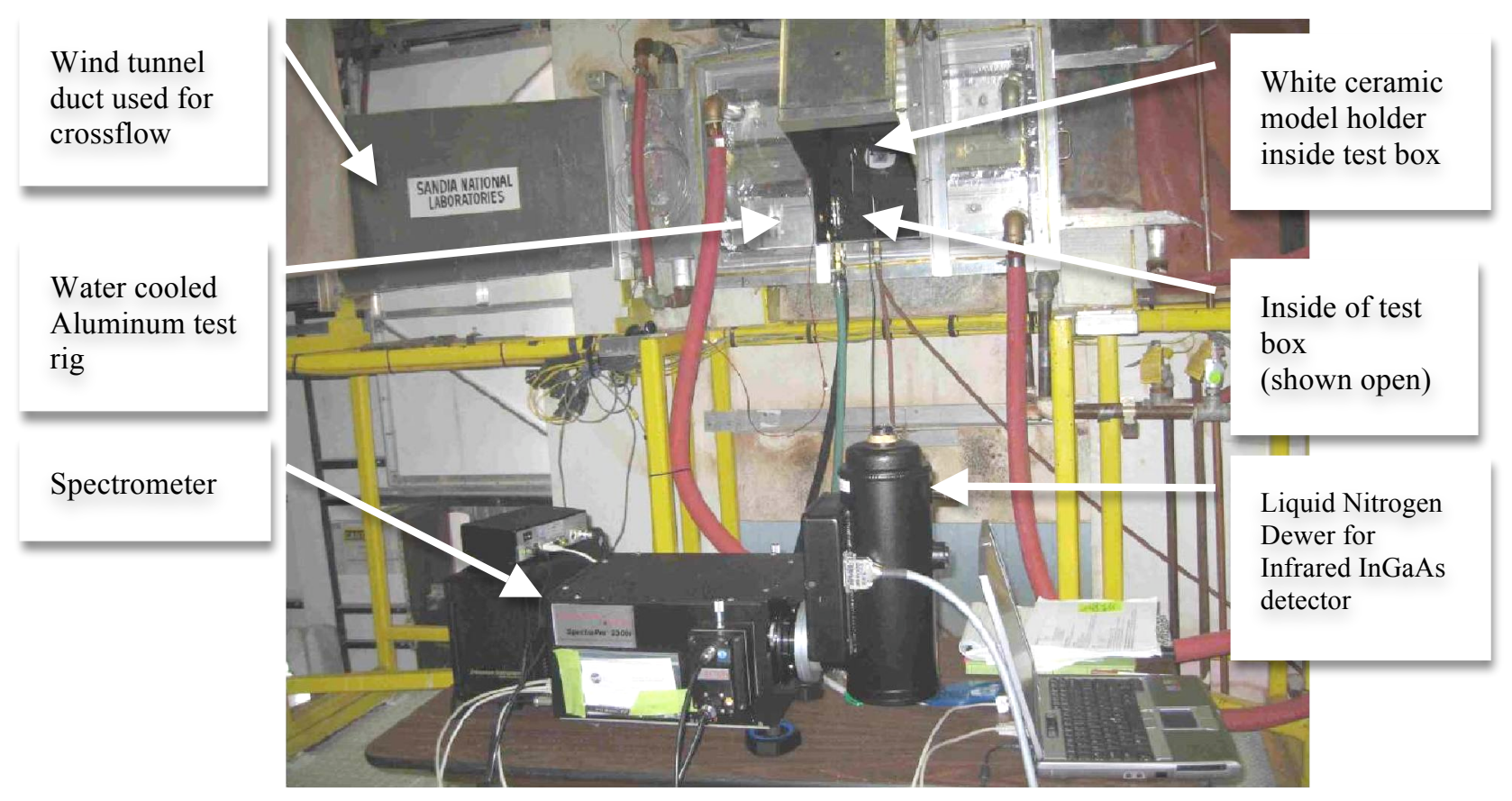

Figure 5. This photograph shows the test box and spectrometer configured for these tests.

Figure 5 shows the open black inside of the test box and the spectrometer used for these tests, configured at the test bay known as the 240 level wind tunnel test bay. This test bay was set up to allow gas cross-flow in a wind tunnel configuration. The test box, painted with Krylon black inside in order to minimize reflections, was ventilated with nitrogen gas flow to limit oxygen reactions, and to control contamination of the optics by pyrolysis products. The test material was inserted into a white ceramic model holder mounted in a water-cooled holder facing normal to the incident concentrated solar radiation beam. The fiber optics and the collimator lens were mounted on a breadboard in the nitrogen gas ventilated test box. The spectrometer and data acquisition system were located out of the direct beam path. This positioning was useful for reducing the strong electrical interference and vibration, for keeping the instruments uncontaminated, and within a good operating temperature range, as well as for safety reasons.

\section{Test Data}

\section{A. Spectral radiation measurements}

The spectral measurements were used to evaluate the potential for direct energy transport by radiation through the 1-10 mm specimens of PICA test materials. For independent measurements to obtain temperature profiles, separate test models with in-depth thermocouples were run to compare to arc jet tests on Phenolic Impregnated Carbon Ablator ${ }^{6}$ (PICA). PICA is a low-density ablative TPS material incorporating a phenolic resin in a low density framework of carbon fibers, which was developed by NASA Ames Research Center staff. PICA has successfully flown on Stardust and was selected for the Mars Science Lander mission. PICA is produced using the preformed fibrous carbon Fiberform material, manufactured by Fiber Materials Inc. (FMI), as the rigid substrate, with phenolic resin as the pyrolyzing material. The Fiberform used in the PICA process is a commercial material used in the insulation industry. The SCI008 phenolic resin is manufactured by Borden and is used in the production of carbon composites. The density of PICA is in the range of 0.224 to $0.248 \mathrm{~g} / \mathrm{cc}$.

The PICA material used in these tests was provided by NASA's Crew Exploration Vehicle Advanced Development Project (CEV ADP) project. PICA was tested at the Solar Tower both in the test configuration described in this paper and in large scale thermostructural tests ${ }^{7}$ on top of the tower. 

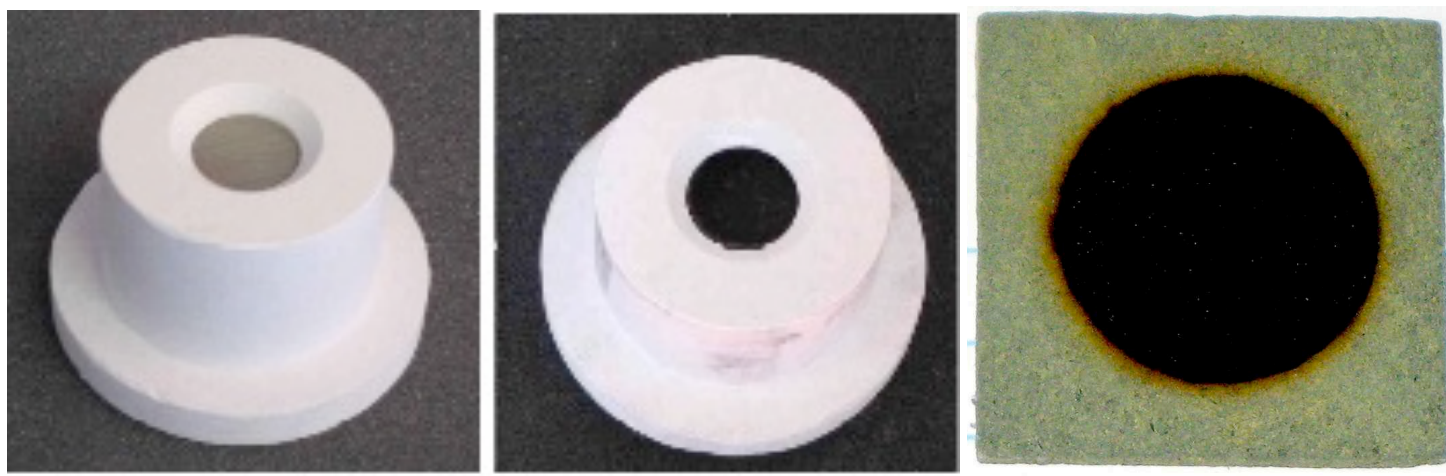

Figure 6. Pre- and post test PICA Sample in model holder and alone (1 sec exposure at $\left.100 \mathrm{~W} / \mathrm{cm}^{2}\right)$

Figure 6 shows the front surface of a thin PICA test sample after exposure at $100 \mathrm{~W} / \mathrm{cm}^{2}$ for one second, with the charred area clearly visible in the magnified photo at the right. The ceramic model holder shielded the unexposed corners of the material during testing. The exposed area diameter was $25 \mathrm{~mm}$ diameter out of a $38 \mathrm{~mm}$ square area. The heat transfer was locally one-dimensional with respect to the one micron wavelength of radiation used, and with respect to the typical $2 \mathrm{~mm}$ test sample thickness. Three-dimensional effects with lateral conduction would strongly affect thermal profiles near the edges, in thicker models, or longer times, however, that was not the focus of these measurements.

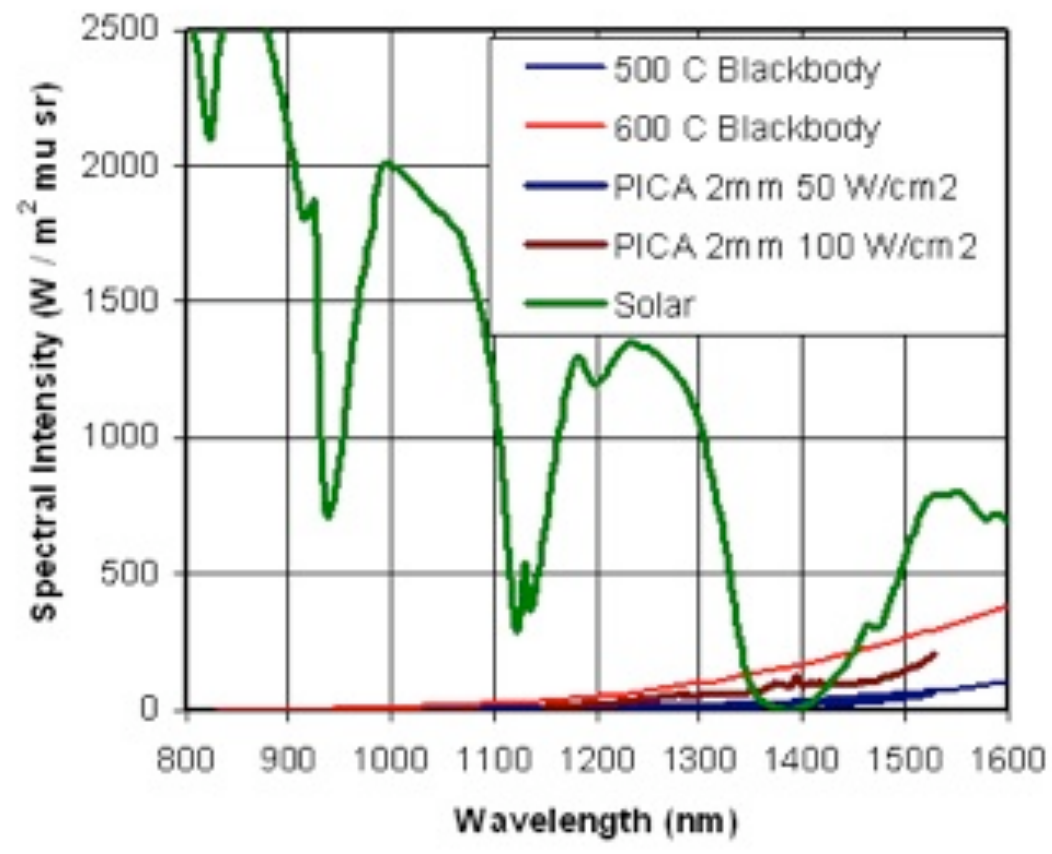

Figure 7. Spectral data as a function of wavelength, with the incident concentrated solar radiation and blackbody radiation intensity shown for comparison.

Figure 7 shows a comparison between the radiant intensity from the classical Planck blackbody spectral distribution $^{8}$ at two temperatures and measured spectral data. The measured spectral data shown are for 2 mm-thick specimens of PICA exposed for one second at the 50 and $100 \mathrm{~W} / \mathrm{cm}^{2}$ levels, and the measured incident concentrated solar radiation. The blackbody curve for the solar temperature would not be useful for comparison, because the terrestrial solar spectrum is strongly influenced by atmospheric absorption in this wavelength region. The curves for the radiant intensity for perfect $500 \mathrm{C}$ and $600 \mathrm{C}$ temperature blackbody emission show idealized blackbody behavior. All measurements on PICA in the wavelength range below $1150 \mathrm{~nm}$ was very low signal dominated by 
noise, and is not shown on the plot. The PICA data for greater than $1 \mathrm{~mm}$ thickness show approximately grey behavior. The spectral data shown above for the heated material gives reasonable grey NIR emittance of 0.80 for the 50 and $100 \mathrm{~W} / \mathrm{cm}^{2}$ exposures.

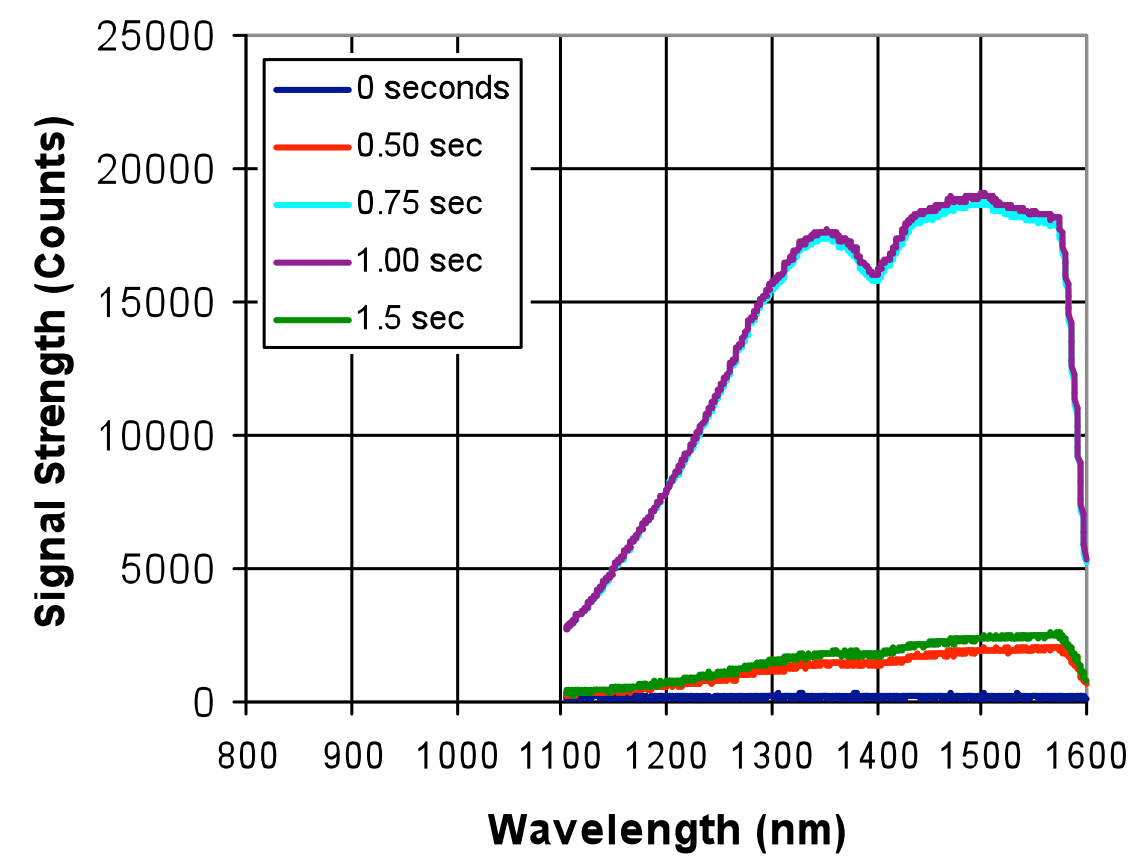

Figure 8. Spectral data for $1 \mathrm{~mm}$ thick PICA as a function of wavelength (1 sec exposure at $50 \mathrm{~W} / \mathrm{cm}^{2}$ )

Figure 8 shows spectral data from $1 \mathrm{sec}$ exposure at $50 \mathrm{~W} / \mathrm{cm}^{2}$ of $1 \mathrm{~mm}$ thick PICA, which showed direct transmission through the pores of the material as well as a thermal radiation profile 0.5 seconds after the shutter doors closed. The $1 \mathrm{~mm}$ PICA showed a sudden increase in transmission between 0.5 and 0.75 second, which is believed to be the time required for the phenolic resin to char. In contrast, as reported elsewhere ${ }^{4}$, the tile materials showed immediate and non-changing transmittance and forward scattering during the exposure, and dropped immediately, without a thermal radiation profile even at 0.5 seconds after the shutter door closed.

In contrast, non-absorbing low-density random refractory fiber materials showed distinct direct spectral transmission and scattering spectra from room temperature laboratory spectrometer measurements as well as during Solar Tower testing ${ }^{4}$. The spectral data from silica fiber based materials such as uncoated shuttle tile materials was consistent with radiation transport through repeated scattering off the fibers. PICA's behavior is consistent with the highly absorbing behavior of pure low density carbon materials ${ }^{9}$ such as soot and carbon fiber materials.

In summary, the spectral data for PICA, for thicknesses greater than $1 \mathrm{~mm}$, showed a spectral profile following the classical Planck blackbody spectral distribution, allowing for experimental error at these very low signal levels, which stands in contrast to the spectral distribution of the solar radiation incident on the test model surface. 


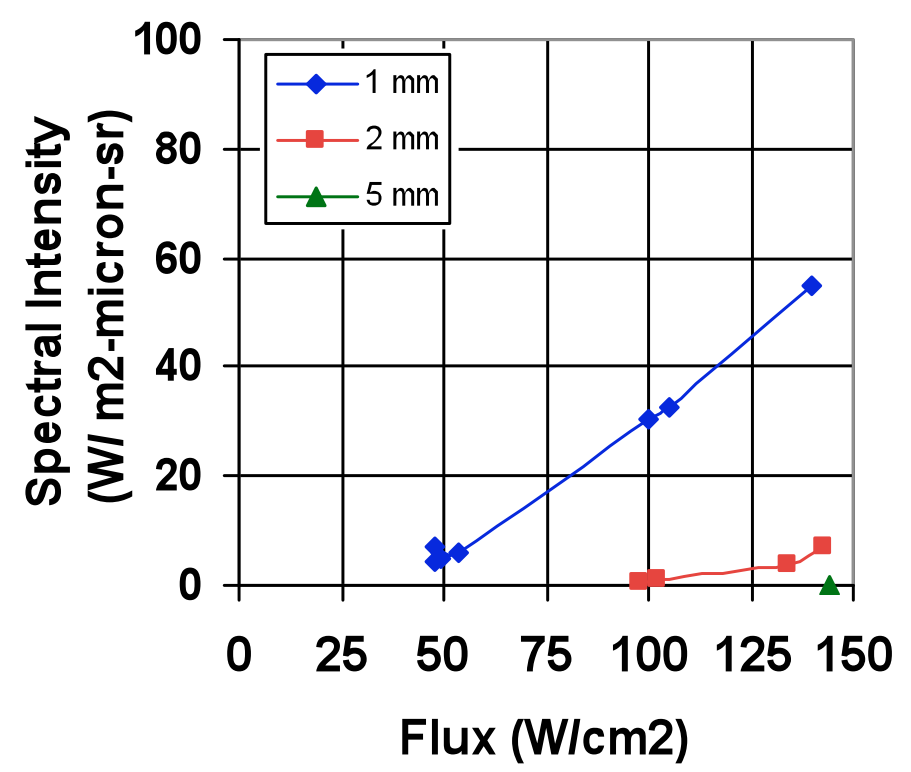

Figure 9. Maximum values of signal strength of the radiation measured at the back face of PICA test models at $1580 \mathrm{~nm}$ for the different test conditions

Figure 9 is a summary of the peak values of the measured signal in detector counts from the PICA test matrix over the 50,100 , and $150 \mathrm{~W} / \mathrm{cm}^{2}$ flux levels attainable with the test setup. An array of material thicknesses (1, 2, 5 and $10 \mathrm{~mm}$ ) was used in order to capture the anticipated nonlinear material response to radiation. This data shows a consistent and reasonable level as well as level of increase in thermal emission with increase in heat load for a fixed thickness or thermal mass, for this changing, pyrolyzing material. The $1 \mathrm{~mm}$-thick samples exhibited limited transmission of the solar spectrum, which is consistent with the porous structure of the material and with possible machining damage, unlike the thicker samples tested. The spectral distributions of the thicker samples showed very low energy levels of blackbody-like profiles consistent with the $2 \mathrm{~mm}$ specimen data.

The PICA backface temperature was computed from the spectral data on the $2 \mathrm{~mm}$ thick models using the Planck distribution; however this is a lower fidelity estimate than a typical pyrometer measurement, since the signal level is low and the wavelength band is focused on a narrow region of interest in these dedicated NIR tests. This NIR range of measurements was relatively far from the peak blackbody wavelengths, e.g. between 3 and 5 microns for temperatures between $700 \mathrm{C}$ and $300 \mathrm{C}$. The PICA backface temperature was measured for some thin models with a backface contact thermocouple, which compared reasonably well with temperatures computed from the spectral data on the $2 \mathrm{~mm}$ thick models, that is, the temperatures derived from the spectral data were within a maximum of $14 \%$ and had an average of $3 \%$ deviation from the temperature in degrees $\mathrm{K}$. The $2 \mathrm{~mm}$ temperatures were within 22 degrees $\mathrm{K}$ of the measured values. The $1 \mathrm{~mm}$ models allow direct transmission and therefore the temperatures did not agree as well, since part of the signal was direct transmission rather than thermal radiation. An alternative approach would use an energy balance to compute temperatures, which would not be limited by contact resistance of a surface thermocouple or by low spectral signal levels, however the convective cooling rate from the gas cross flow was unknown, making this approach tunable by changing the cooling rate but inaccurate. Since that was not the focus of these tests, that approach was not pursued further.

\section{B. Thermocouple measurements}

Thermocouple measurements were made to evaluate the material's response to radiation, independent of the spectral measurements. 


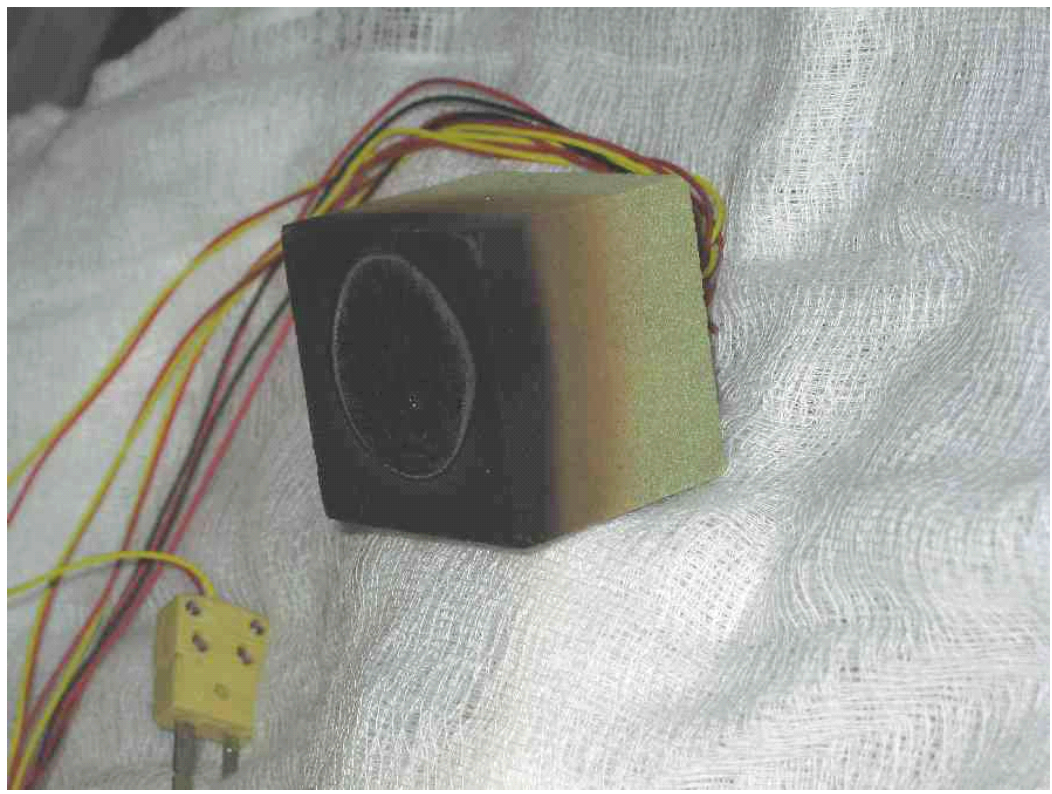

Figure 10. Thermocouple instrumented model of PICA after exposure in the Solar Tower tests

Figure 10 is a photograph of a test model of PICA instrumented with a standard thermocouple plug after exposure in the Solar Tower tests. The char and pyrolysis zones are clearly seen on the sides, which were insulated by the ceramic holder, but heated due to the relatively high in-plane thermal conductivity of PICA. Comparing the in-depth temperature profiles from radiative heating versus convective arc-jet heating tests allowed a separate evaluation of thermal response. In-depth temperatures were measured using accurately positioned thermocouples in a standard plug configuration, and showed thermal soak trends consistent with diffusion-controlled thermal transport. Specifically, the measured in-depth temperatures did not exhibit the red flag of a sudden rise or step, either initially or during charring, which would have corresponded to undesirable rapid transmission of radiation through the test material.

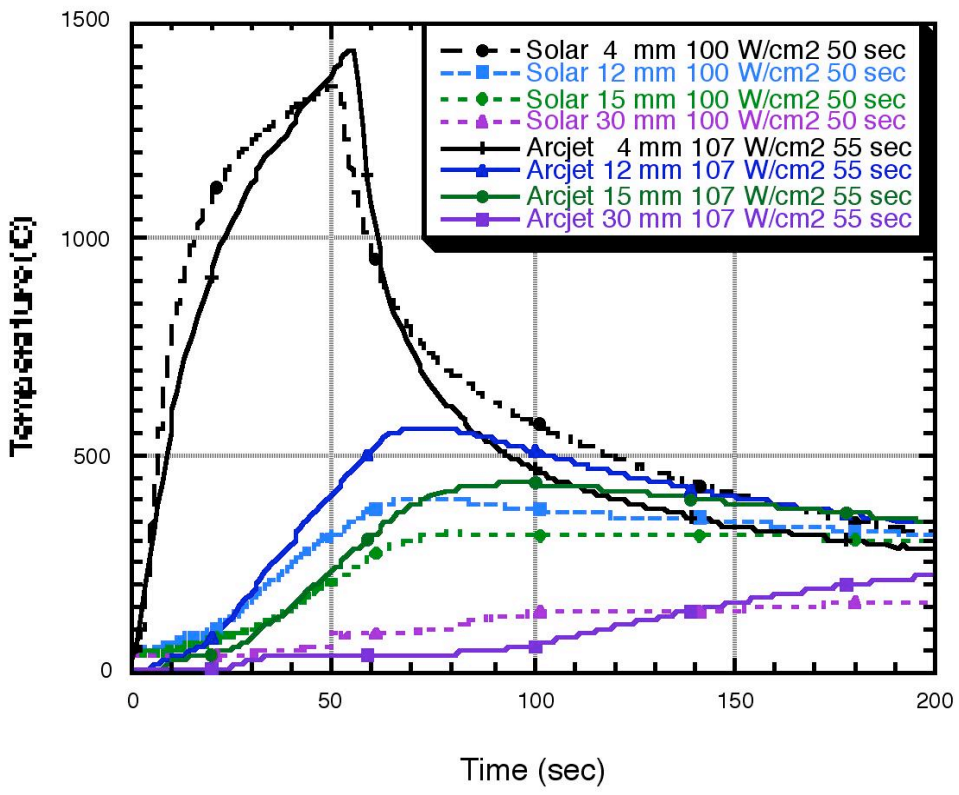

Figure 11. Temperatures are plotted against time for in-depth thermocouples, showing the thermal response to radiation heating for $100 \mathrm{~W} / \mathrm{cm}^{2}$ for 50 seconds and to convective arc-jet heating of $107 \mathrm{~W} / \mathrm{cm}^{2}$ for 55 seconds. 


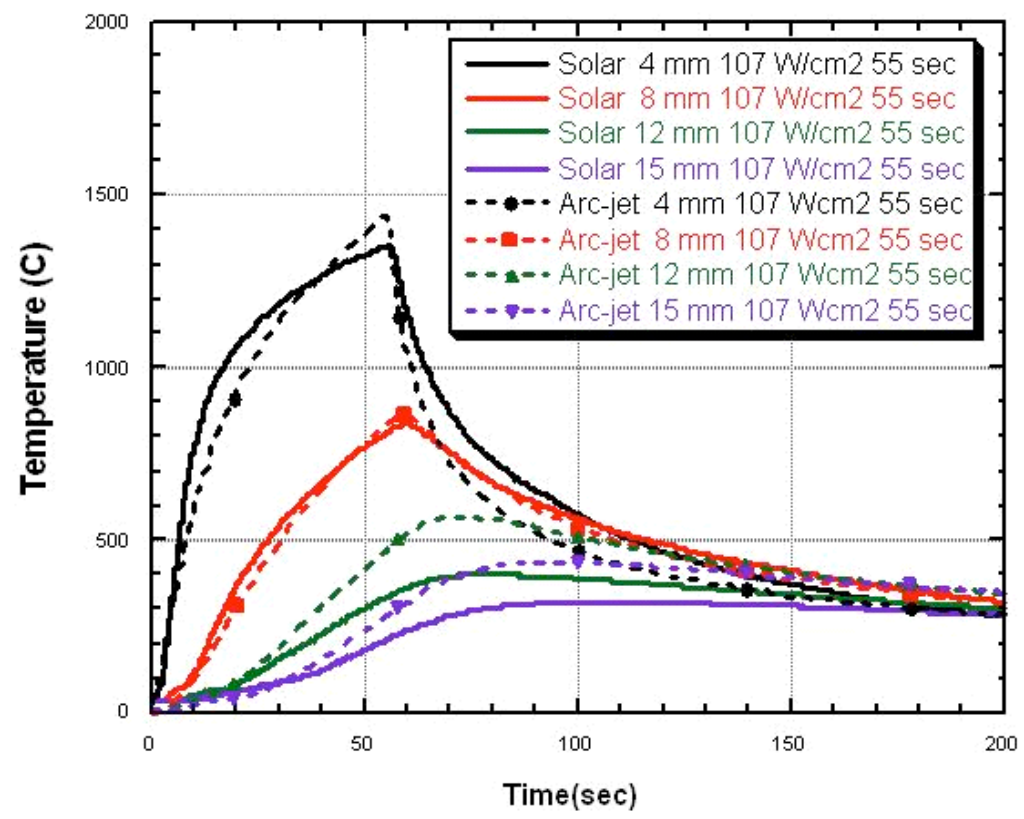

Figure 12. Temperatures are plotted against time for in-depth thermocouples, showing the thermal response to radiation heating and to convective arc-jet heating for $107 \mathrm{~W} / \mathrm{cm}^{2}$ for 55 seconds exposures

Figures 11 and 12 show the solar radiation thermocouple data plotted with the most comparable arc-jet test data. The arc-jet data is for similar heat load, flux, and duration of $107 \mathrm{~W} / \mathrm{cm}^{2}$ for 55 seconds, compared to the Solar Tower radiation tests. The Solar Tower radiation tests were run at the test conditions of $107 \mathrm{~W} / \mathrm{cm}^{2}$ for 55 seconds as well as from an exposure of $100 \mathrm{~W} / \mathrm{cm}^{2}$ for 50 seconds. The lower flux was used to compensate for the hot wall calorimeter correction for the arc jet cold wall calorimeter. These models did not have identical instrumentation depths. The imposed fluxes are not equivalent even for the $107 \mathrm{~W} / \mathrm{cm}^{2}$ for 55 seconds cases, due to the reduction of heat transfer by blowing effects and calorimeter corrections in the arc-jet test, as well as due to surface cooling by the imposed cross flow of nitrogen gas in the solar test. Close agreement is seen at the 4 and $8 \mathrm{~mm}$ depth thermocouple profiles near the top surface, during both heat up and cooldown phases. Reasonable agreement is also seen during the initial heat pulse even at lower depths. The solar test model exposed at $100 \mathrm{~W} / \mathrm{cm}^{2}$ for 50 seconds included a $30 \mathrm{~mm}$ depth thermocouple, which showed the greatest divergence between the solar and the arc-jet data.

The Solar Tower radiation test data and convective arc-jet are seen to agree very well in the regimes where the temperature field is nearly 1-dimensonal: in the thermocouples nearest the top surface, and during the initial heat pulse. However, at greater depths or over longer times, close agreement between the radiation test data and arc jet test data would not be expected for several reasons. At greater depths and longer times, temperature differences are dominated by geometric 3-dimensional effects as well as thermal mechanisms. The geometric effects include the unheated side walls in the radiation test model versus than in the side wall heating in the arc-jet test model, and the more limited exposure area of $25 \mathrm{~mm}$ diameter in the $38 \mathrm{~mm}$ square front area in the radiation test model leaving virgin material at the sides, versus the fully exposed arc-jet test model front face. These effects are exacerbated by lateral in-plane conductivity effects. In addition, PICA's thermal response in these tests is influenced by differences between the ablation mechanisms themselves due to the gas composition and pressure, as well as by surface cooling by the imposed cross flow of nitrogen gas, and blowing effects in the arc-jet test.

Milos and Chen ${ }^{10}$ have shown excellent agreement can be obtained for PICA models between the measured indepth thermal response of PICA to convective arc-jet heating and numerical modeling using FIAT and TITAN. For axisymmetric or nearly 1-dimensional PICA models, computed temperature profiles closely track the measured arcjet measurements along the centerline. To get reasonable in-depth agreement with radiation test data, however, more axisymmetric or 1-dimensional radiation test models than those used in these tests are needed.

In summary, the in-depth temperature profiles show graphically that under these test conditions, PICA's thermal response is similar whether PICA is subjected to pure radiative heating, as in the Solar Tower tests reported here, or pure convective heating, as in arc-jet tests. The spectral data showed that PICA maintains negligible direct transmission and responds in a diffusion dominated manner. Because it is optically thick, PICA responds similarly 
to incident radiation as it responds to imposed convection. There were no red flags indicating direct transmission and in-depth absorption, which would be detectable as sudden steps up of the in-depth temperatures, and sudden steps down when the shutter doors closed to cut off the incident radiation pulse.

The spectral data and the thermocouple measurements independently support the conclusion that the PICA acts as a surface absorber, with very small extinction lengths.

The overall behavior of the in-depth temperature profiles is that of a classical diffusion-dominated temperature field ${ }^{11}$, indicating that PICA response to radiation can be used to back out an effective combined radiation and thermal diffusion coefficient, and/or an effective combined radiation and thermal conductivity.

\section{Modeling Radiation transport}

PICA's radiative transport behavior and the data presented here can be used to better understand and extrapolate the relationship between the conductivity property values measured under steady state, low-temperature gradient conditions such as guarded hot plate values, and the dynamic values extracted from high-temperature gradient arcjet data, as well as to improve thermal modeling of PICA.

PICA's response to pure radiation is consistent with radiation transport theory for strongly absorbing materials such as carbon even for low density porous absorbing materials. The diffusion approximation can be used in the limit of optically thick materials like PICA. The diffusion approximation predicts an effective radiative diffusivity analogous to thermal diffusivity. Alternatively to measuring the effective diffusion coefficient, rigorous modeling of radiation transport through low-density fiber materials ${ }^{12}$ has been developed, including absorption with scattering through random fibrous materials with an alternate solid phase present between the fibers. Solving for radiation transport from first principles is computationally intensive, incorporating the complex geometry of the quasirandomly oriented fibers, and can be limited by the availability of the fundamental temperature-dependent transport properties of the components.

Radiation energy transport follows different physical laws than convective or conductive energy transport, and properties can be wavelength- as well as temperature- and composition- dependent, therefore ablative TPS, like other materials, can respond to radiation heat transfer very differently than to convection.

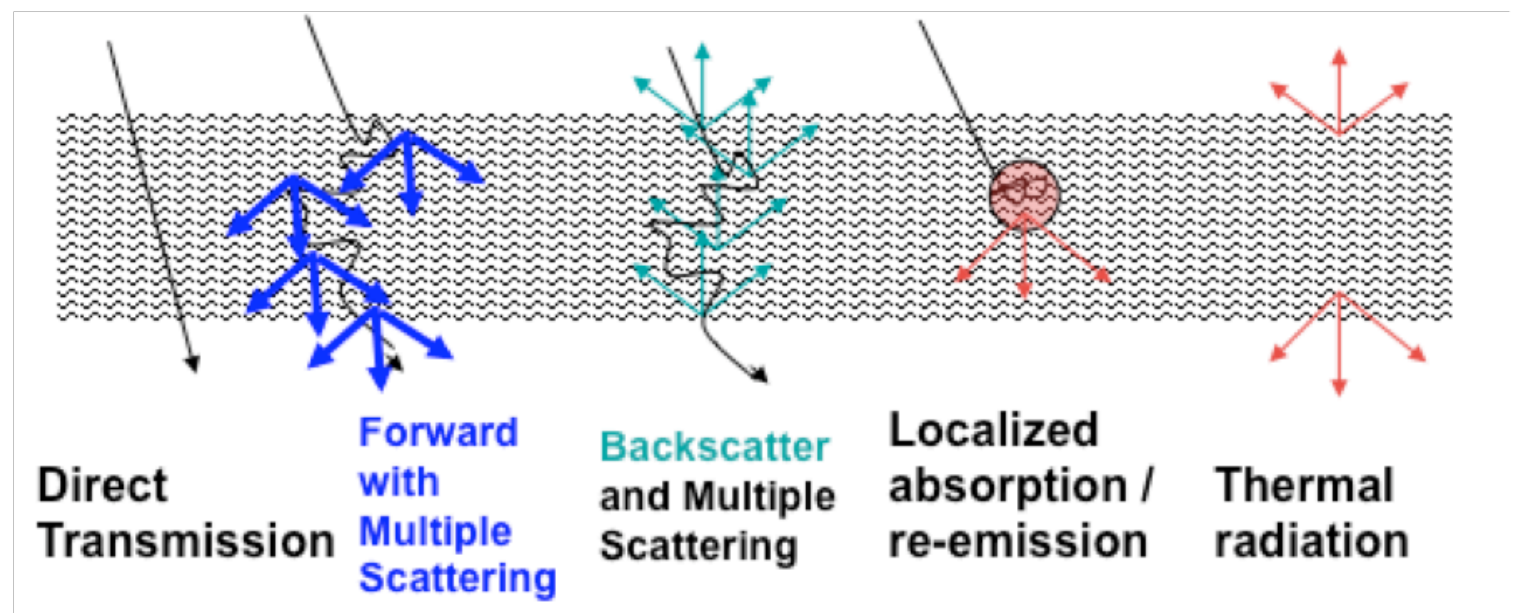

Figure 13. Radiation transport through a scattering porous material.

Figure 13 is a schematic shows radiation transport including scattering, emission etc. occurring in porous fibrous tile and carbonaceous fiber or char. The repeated scattering and local absorption changes the transport through the material. In-depth absorption of radiation energy causes a temperature increase, which is reduced by radiation emission. Emission will cause a wavelength distribution similar to blackbody radiation. Forward scattering of the incident radiation occurs at every scattering interface, and changes the direction of energy flow, but does not change the wavelength distribution. Radiation must be integrated over all wavelengths and solid angles for hemispherical total values generally used in thermal computations. 
Radiation transport ${ }^{8}$ is governed by the combined integral differential radiative transfer equation, which offers a formidable challenge to solution in many cases. The radiative transfer equation for the spectral intensity $i_{\lambda}$ as a function of the opacity or optical depth $\mathrm{d}_{\lambda}$, is given below in terms of the source function I. The source function I includes the scattering phase function $\Phi$, which depends on the scattering materials geometry and properties. When scattering $\sigma$ is present, I is expressed most directly with the albedo $\Omega$, which is the scattering coefficients divided by extinction coefficient.

$$
\begin{aligned}
& \frac{d i_{\lambda}}{d d_{\lambda}}+i_{\lambda}=I_{\lambda}\left(d_{\lambda}, \omega\right) \\
& I_{\lambda}\left(d_{\lambda}, \omega\right)=\left(1-\Omega_{\lambda}\right) i_{\lambda b}\left(d_{\lambda}\right)+\frac{\Omega_{\lambda}}{4 \pi} \int_{0}^{4 \pi} i_{\lambda}\left(d_{\lambda}, \omega_{i}\right) \Phi\left(\omega, \omega_{i}\right) d \omega_{i} \\
& \Omega_{\lambda}=\frac{\sigma_{s \lambda}(\lambda, s)}{\kappa_{\lambda}(\lambda, s)}=\frac{\sigma_{s \lambda}(\lambda, s)}{a_{\lambda}(\lambda)+\sigma_{s \lambda}(\lambda, s)}
\end{aligned}
$$

The radiative transfer equation has been directly solved in closed form for certain limiting cases, and it has been solved numerically for a number of very well defined cases with appropriate and completely defined geometry and completely known properties. Specifically, complete knowledge would be required of the locations and orientations of the fibers, the resin, with temperature, composition, as well as the temperature- and wavelength-dependent radiative transport properties, or the complex index of refraction, known at each location.

Energy transfer by radiation is included in the energy transport equation as a divergence term, similar to energy transport by conduction. The radiation flux at a given location, $\mathrm{s}$, is the integrated sum of all wavelengths and depends on the opacity or optical depth $\mathrm{d}$, the absorption a, and the Source function I integrated over the solid angle $\omega$.

$$
\nabla \cdot q_{r a d}(s)=4 \pi \int_{0}^{\infty} \frac{a_{\lambda}\left(\lambda, d_{\lambda}\right)}{\Omega_{\lambda}\left(\lambda, d_{\lambda}\right)}\left[i_{\lambda b}\left(d_{\lambda}\right)-\int_{0}^{4 \pi} I_{\lambda}\left(d_{\lambda}, \omega\right) d \omega\right] d \lambda
$$

The scattering, extinction and absorption coefficients have units of inverse length and each coefficient has a dimensional inverse, scattering, extinction and absorption lengths, which have units of length. The optical depth, d, is the dimensionless distance computed by integrating the extinction or other coefficient over the physical length.

$$
\begin{aligned}
& \kappa_{\lambda}=a_{\lambda}+\sigma_{s \lambda} \\
& d_{\lambda}(\lambda, s)=\kappa_{\lambda}(\lambda, s) * s=\int_{0}^{s}\left[a_{\lambda}(x)+\sigma_{s \lambda}(x)\right] d x
\end{aligned}
$$

The Planck mean absorption coefficient is based on local blackbody radiation.

$$
a_{P}(T, P)=\frac{\int_{0}^{\infty} a_{\lambda}(\lambda, T, P) e_{\lambda b}(\lambda, T) d \lambda}{\int_{0}^{\infty} e_{\lambda b}(\lambda, T) d \lambda}=\frac{\int_{0}^{\infty} a_{\lambda}(\lambda, T, P) e_{\lambda b}(\lambda, T) d \lambda}{\sigma T^{4}}
$$

The extinction coefficient computed from the spectral data measured in the Solar Tower tests gives an extinction coefficient of $7.7 \mathrm{~mm}^{-1}$, and an extinction length of $0.13 \mathrm{~mm}$. This compares closely to the computed extinction length: Radiation transport coefficients were computed from first principals by S.C. Lee ${ }^{7}$ for a carbon fiber matrix, 
to simulate radiation transport through the FMI carbon fiberform, the structural framework of PICA, without resin loading, with 12 micron diameter fibers loosely packed to a density of $0.1 \mathrm{~g} / \mathrm{cc}$. The complex index of refraction for carbon used was from Howarth and Foster. The extinction coefficient asymptotes to $90 \mathrm{~cm}^{-1} \mathrm{or}^{\mathrm{mm}} \mathrm{mm}^{-1}$, which corresponds to an extinction length of $0.11 \mathrm{~mm}$.

Given more time and resources, the divergence term and the radiation transport equation for this quasi-random absorbing and scattering medium could be solved directly with high-fidelity numerical models built on models and experiments for fibrous materials ${ }^{8}$ to compute the required transmission and absorption. Lower fidelity approximations can be built on the substantial body of useful approximations and existing knowledge $\mathrm{e}^{5,8}$, for example, using existing numerical solutions for planar 1-dimensional scattering absorbing layers, and using the Stark conduction-radiation parameter or scattering efficiencies. An extensive literature is available on carbon particles, which could be exploited to model the char layer radiation transport properties in terms of carbon particle volume fraction developed for combustion and flame applications, however it is more appropriate to smaller size parameter, more symmetrical, and lower density materials. These approaches are also beyond the scope of the current effort.

The powerful diffusion approximation is a robust, direct and productive approach to simplify this problem. The radiation diffusion approximation is valid for optically thick materials, and allows radiation transport to be modeled as a decoupled diffusion-like term in the energy equation. Radiation diffusion theory uses an effective radiative diffusivity or radiative conductivity within an optically thick material. The radiation diffusion model is not strictly correct at the material boundaries, and alterative computational methods or radiation slip approaches are required and have been developed to include the boundaries. Energy transport governed by radiative diffusion is decoupled from molecular or thermal transport and ultimately results in an increase in the overall effective diffusivity or conductivity, increasing energy transfer within and through the material.

To evaluate useful transport properties, effective total mean properties must be used, which depend on position, composition, temperature, and history. This approach uses approximations to avoid solving the radiative transport equation for the specific composite material to include scattering from first principals or fundamental complex refractive index properties. In addition, this approach does not resolve the boundaries, or precisely account for the changing material composition, but it is a practical approach, given the very challenging and computationally intensive alternative of solving the radiative transfer equation for this complex and changing scattering composite material. Ideally, this would be verified against exact solutions.

Radiation diffusion incorporates the materials spectral variation through the extinction coefficient, or by its inverse, the extinction length. The effective mean extinction coefficient is evaluated by integrating the wavelength dependent extinction or its inverse, weighted by the appropriate spectral intensity. An ideal non-scattering material would have an extinction coefficient independent of thickness and history, and it depends on the temperaturehistory-dependent composition. Multiple dependent scattering is intrinsically thickness dependent because of the interaction between scatterers, here primarily the fibers. This inconvenient fact has been shown theoretically for scattering fiber media. In addition, the material itself changes rapidly during testing with heating and charring: the different thicknesses of the materials developed different percentages of char vs. virgin zones, as well as temperatures, so upon heating the different thicknesses tested were in fact of different and rapidly changing compositions.

For incident shock layer radiation for lunar return, only the scattered component of the radiation from the shock layer radiation distribution in the near infrared will maintain the incident spectral distribution of the incident beam, whereas the absorbed and emitted radiation will be distributed predominantly over longer infrared wavelengths, following the Planck blackbody thermal radiation distribution for the local temperature weighted by the emittance. Ideally, the scattering and absorption would be resolved from first principals from the radiative transport equation, but using effective diffusion coefficients allow rapid and efficient modeling.

The ratio of dimensionless diffusivity ratio was defined to simplify computations and tabulate a modified total conductivity, combining the radiative diffusive transport with thermal conductive transport, for thermal modeling. 


$$
\begin{aligned}
& k_{\text {radiation }}=\frac{1}{\kappa_{\text {eff }}}\left[\frac{16 \sigma T^{3}}{3}\right]=l_{\text {eff }}\left[\frac{16 \sigma T^{3}}{3}\right] \\
& q^{\prime \prime} \equiv\left[\frac{16 \sigma T^{3}}{3 \kappa_{\text {eff }}}+k_{\text {thermal }}\right] \nabla T \equiv\left[k_{\text {radiation }}+k_{\text {thermal }}\right] \nabla T \\
& \quad=\rho c_{p} \alpha_{\text {thermal }}\left[\frac{\alpha_{\text {radiation }}+\alpha_{\text {thermal }}}{\alpha_{\text {thermal }}}\right] \nabla T
\end{aligned}
$$

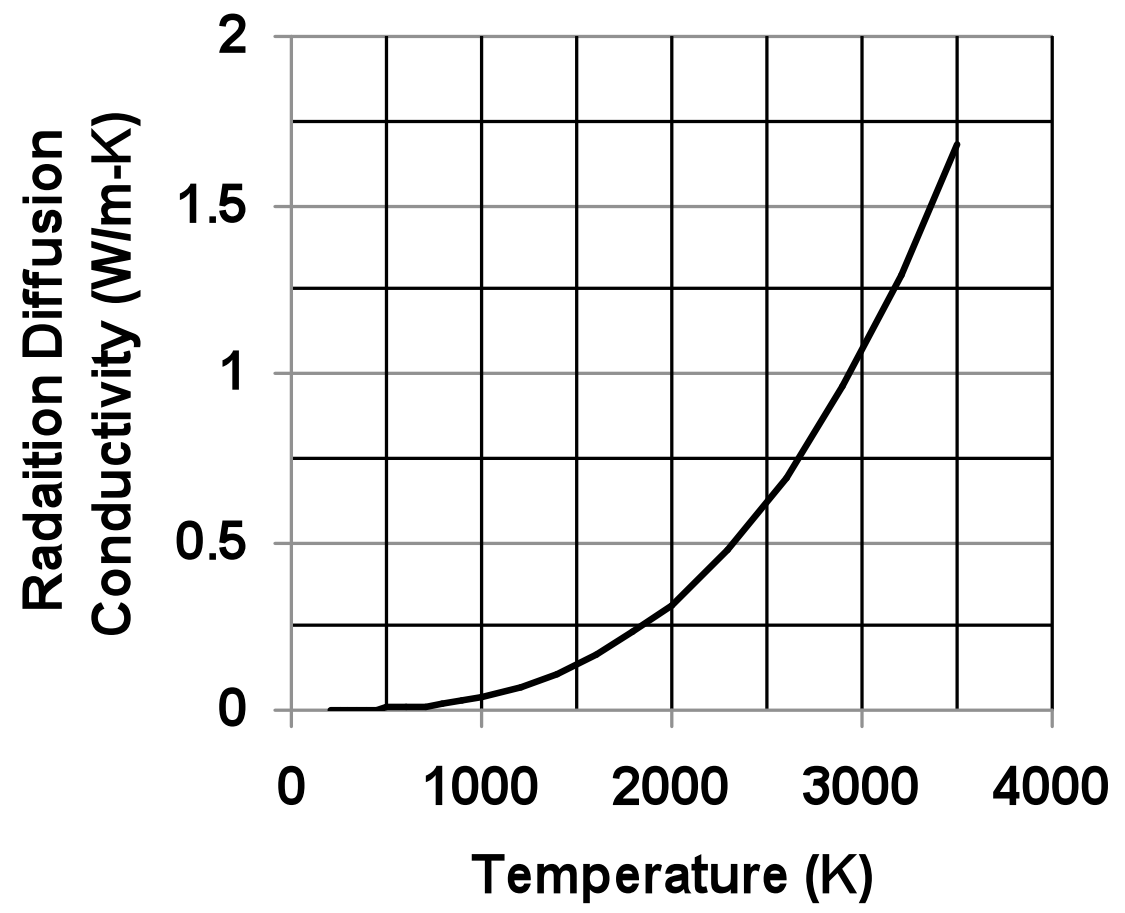

Figure 14 shows the computed radiative conductivity as a function of temperature based on the diffusion approximation.

Figure 14 shows the radiative conductivity for PICA, computed using the wavelength dependent spectral transmission data for the diffusion approximation. The diffusion model gives a reasonable temperature dependent trend for this radiation effect, which can be used to evaluate and adjust the thermal conductivity measured under steady state conditions, to compare it to the dynamic conductivity measured under high temperature gradient conditions such as arc-jet heating. 


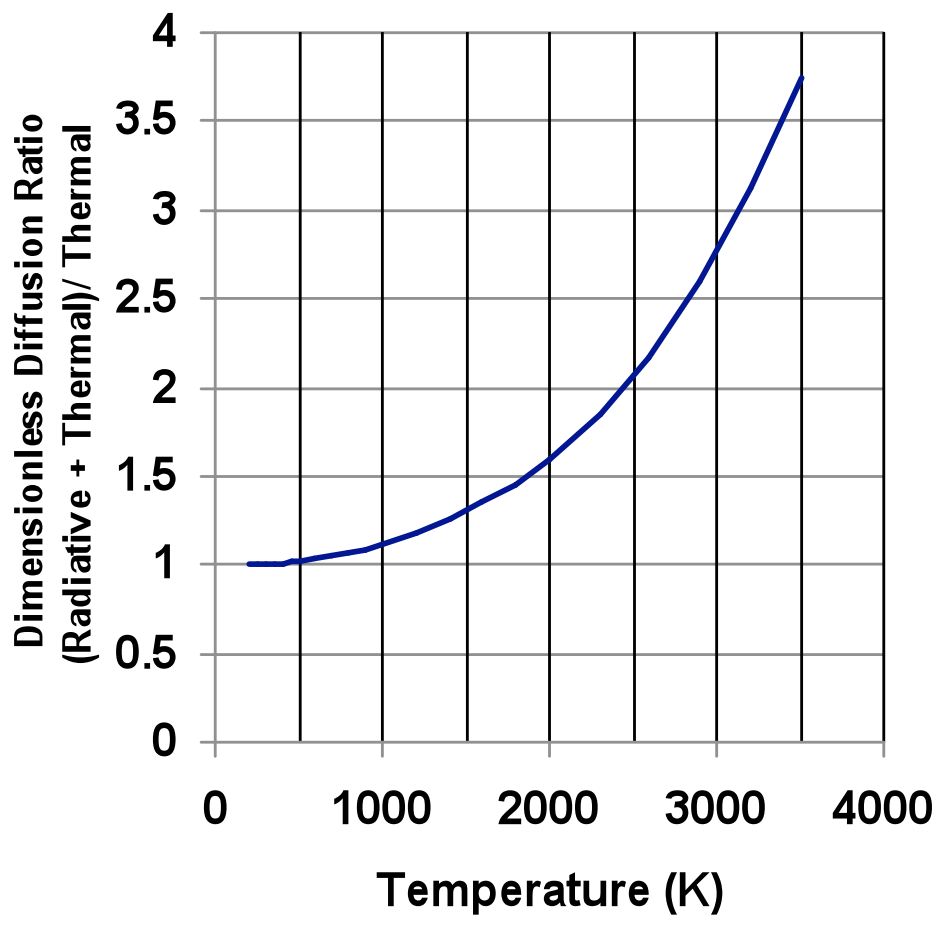

Figure 15 shows the dimensionless ratio of the total effective combined radiative and thermal diffusivity to the thermal diffusivity illustrating the scaled magnitude of radiation diffusivity.

Figure 15 shows the magnitude of the effect of including radiation in thermal modeling giving the dimensionless ratio of the total effective combined radiative and thermal diffusivity to the thermal diffusivity. The diffusion model gives a reasonable temperature dependent ratio of effective diffusivity to the thermal diffusivity. The wavelength dependent spectral transmission data was used to compute an effective extinction coefficient $\kappa_{\text {eff }}$ and to evaluate the dimensionless ratio of the total effective combined radiative and thermal diffusivity to the thermal diffusivity.

In summary, PICA is an optically thick porous material, in which thermal diffusion is influenced by radiation diffusion effects. Radiation transport can be included in or excluded either from thermal diffusivity property values or from the energy transport equation in a straightforward manner via the diffusion approximation. In general, radiation transport has a greater effect when the absolute temperature and temperature gradients are high, proportional to the third or fourth power of the absolute temperature depending on the specific temp of interest. For an optically thick material such as PICA, the thermal diffusivity evaluated from dynamic high temperature and high temperature gradient test conditions is an effective combined diffusivity which already includes the radiative diffusivity component. Steady state, low temperature measurements would not include a significant radiation transport component.

Ground test and small sample set caveats: The impact of radiation transmission on flight performance may be different than measured in ground tests, because Apollo flight chars were different than chars produced in ground tests (e.g, higher density, lower porosity, higher carbon content due to char "coking"). Higher density, lower porosity coked chars should reduce radiant transmission due to increased opacity. Preliminary evaluation of the mean coefficient indicates that the extinction is high enough that PICA acts as a surface absorber within the top few millimeters such that significant radiation does not penetrate in-depth. For future use in higher radiation environments, higher fidelity numerical modeling and higher flux experiments are recommended. Higher fidelity numerical modeling of fiber scattering and the resin would allow rigorous inclusion of scattering from fibers from first principles and would guide extrapolation outside the envelope of ground tests. Further testing would be useful using higher fluxes at the Solar Tower testing, NIR laser, on virgin and charred material, using vacuum baked, and if possible coked, models, in order to generate sufficient data to rigorously include radiation transport in modeling. Sufficient repetitions should be run to confidently and accurately evaluate the radiation transport. Larger scale thermocouple-instrumented models should be tested in order to reduce 3-dimensional effects, at the Solar Tower and 
using the Wright-Patterson 1.07 micron CW fiber laser. Using data from larger test models, the overall effective diffusivity ratio can be independently evaluated from scaled experimental data using the Boltzman transformation and compared to diffusion theory. The scaled experimental data would be a useful reality check to ground the diffusion theory computations.

\section{V.Conclusions}

Both spectral measurements and measured in-depth temperature profiles showed that, although it is a porous, low-density material, PICA functioned in these tests as a surface absorber with negligible transmittance at the tested high levels of NIR radiation. PICA acted as a surface absorber to efficiently absorb the incident visible and near infrared incident radiation in the top 2 millimeter layer in the Solar Power Tower tests up to $150 \mathrm{~W} / \mathrm{cm}^{2}$. Thin $1 \mathrm{~mm}$ samples showed transmission, but the thickness scaled by the pore size was not large enough to consider a $1 \mathrm{~mm}$ sample to be homogeneous.

The measured in-depth PICA temperature profiles support the concept that PICA responds similarly to radiation as to convection. The thermal transport mechanisms and effective thermal diffusivity in PICA are similar whether PICA is subjected to pure radiative heating, as in the Solar Tower tests reported here, or pure convective heating, as in arc-jet tests. This is consistent with radiation transport theory, which allows the simplified diffusion approximation for optically thick materials, and is consistent with strong extinction due to the efficiently absorbing carbon fiber components in PICA. Energy transport in PICA by radiation transport effects can be included in modeling using radiation diffusion models.

\section{Acknowledgments}

This work was supported by and performed for NASA's Orion Crew Exploration Vehicle Advanced Development Project. The author would like to thank many people for useful technical discussions and collaboration, including Jim Arnold, Dave Bogdanoff, Y.K. Chen, Joe Conley, Brett Cruden, Don Ellerby, Jay Grinstead, Bernard Laub, Frank Milos, George Raiche, James Reuter, and Ethiraj Venkatapathy of NASA Ames Research Center, Siu-Chun Lee of Applied Sciences, and the Solar Power Tower staff members Cheryl Ghanbari, J.J. Kelton, and Daniel Ray of Sandia National Laboratory.

\section{References}

\footnotetext{
1 Bose, D., McCorkle, E., Thompson, C., Bogdanoff, D. W., Prabhu, D., Allen Jr., G. and Grinstead, J., “Analysis and Model Validation of Shock Layer Radiation in Air", AIAA 2008-1246.

2 Bogdanoff, D. W., NASA Ames Research Center, personal communication.

3 Quijada, M.A., NASA Goddard Space Flight Center, personal communication.

4 White, Susan, "High-Temperature Spectrometer for Thermal Protection System Radiation Measurements", Jnl. Spacecraft and Rockets, Vol. 47, No. 1, 2010, pp. 21-28.

5 Cruden, B.A., Martinez, R., Grinstead, J. and Olejniczak, J., "Simultaneous Vacuum Ultraviolet through Near IR Absolute Radiation Measurement with Spatiotemporal Resolution in an Electric Arc Shock Tube", AIAA Paper 2009-4240, June 2009.

6 Tran, H., Johnson, C., Rasky, D., Hui, F., Chen,Y.-K., and Hsu, M., "Phenolic Impregnated Carbon Ablators (PICA) for Discovery Class Missions," AIAA Paper 96-1911, June 1996.

7 Agrawal, P., Empey, D.M., and Squire, T.H., "Thermal-Structural Analysis of PICA Tiles for Solar Tower Test," AIAA Paper 2009-3755, June 2009.

8 Siegel, R. and Howell, J.R., Thermal Radiation Heat Transfer, 2nd ed., McGraw-Hill, New York, 1981, Sec. 8-4.

9 Eckert, E.R.G., Tien, C.L., and Edwards, D.K., "Radiation," Handbook of Heat Transfer Fundamentals,2nd ed., edited by Rohsenow, W.M., Harnett, J.P. and Ganik, E.N., McGraw-Hill, New York, 1985, Chap. 14.

10 Milos, F.S. and Chen, Y.K., "Ablation and Thermal Response Property Model Validation for Phenolic Impregnated Carbon Ablator”, AIAA paper 2009-262, Jan. 2009.

11 Bird,R.B., Stewart, W.E. and Lightfoot, E.N., Transport Phenomena, 2nd ed.,Wiley, N.Y.,2007 Sec 16.5 and 20.1, pp.502507 and 613-622.

12 Lee, S.C., White, S.M., and Grzesik, J., "Effective Radiative Properties of a Fibrous Composite containing Spherical Particles", AIAA Jnl. Thermophysics, Vol. 8, No. 3, 1994, pp. 400-405.
} 